\title{
A Comparative Evaluation of Rotator Cuff Injuries of the Shoulder Joint using High Resolution Ultrasound and Magnetic Resonance Imaging
}

\author{
Shrestha MS', Alam A ${ }^{2}$ \\ ${ }^{1}$ Consultant Radiologist, Shree Birendra Hospital, Chhauni, ${ }^{2}$ Department of Radiodiagnosis, Armed Forces Medical \\ College, Pune
}

\begin{abstract}
Introduction: Pathology of the rotator cuff is the cause of most common problems at the shoulder joint and accurate diagnosis is essential for appropriate management. High-resolution real-time ultrasonography and MRI are successful imaging modalities for both rotator cuff and non-rotator cuff disorders and have important implications in the management of rotator cuff pathologies. This study was conducted to Compare the results obtained after the evaluation of rotator cuff injuries of shoulder joint by high resolution Ultrasonography and Magnetic Resonance Imaging.

Methods: 50 patients with suspected rotator cuff injuries of the shoulder joint were included in this study over a period of 2 years. Patients had presented with various complaints like prolonged pain in the shoulder joint with stiffness,restriction and difficulty in movement of the shoulder joint, traumatic injuries and recurrent dislocations of the shoulder joints.

Results: Mean age was 41.6years ( range 15-80).Maximum patients were between 41-50 years (24\%). 29 were males and 21 were females. In our study diagnostic accuracy of ultrasound in rotator cuff tears was $57.14 \%$ for full thickness tears and 58.33 for partial thickness tears. The diagnostic accuracy of ultrasound in detection of complete tear of the supraspinatus muscle was $57.14 \%$ while that of MRI was $100 \%$.

Conclusion: High resolution Ultrasound being a dynamic study can be used as the first-line investigation for rotator cuff tear. Full-thickness rotator cuff tears can be identified using ultrasound and MRI with comparable accuracy.
\end{abstract}

Key words: High Resolution Ultrasound, Magnetic Resonance Imaging, Rotator Cuff

\section{Introduction}

Rotator cuff injuries are described as partial and full thickness, acute and chronic, traumatic and degenerative. Cuff failure is almost always peripheral near the attachment of the cuff to the tuberosities and always begins in the supraspinatus part of the rotator cuff near the biceps tendon'.

Rotator cuff failure is one of the most common causes of the shoulder pain and dysfunction in patients over 40 years of age. Fiber failure demonstrates a sequential progression from the partial thickness tear almost always starting in the Supraspinatus to massive tears involving multiple cuff tendons ${ }^{2}$. A full thickness tear involves tendon defect, which extends all the way from articular to the bursal surface of the rotator cuff and a partial thickness tear involves only the superficial surface, mid substance or deep surface. Acute tears occur suddenly as a result of a definite injury, whereas

Address for Correspondence:

Name: Dr. Mukunda Singh Shrestha

E-mail: shrestha_mukunda@hotmail.com

Ph. No.: 9841444004 
chronic tears are those that have existed for a long time, eg three months or more and may be insidious in onset and degenerative in nature.

The introduction of Ultrasound machine with highresolution linear array transducers has greatly expanded the diagnostic capabilities in the examination of shoulder. Ultrasound is effective in the detection of acute traumatic tears of the rotator cuff. Transducers with frequency of 7 to $10 \mathrm{MHz}$ and even higher frequency are preferable to those with lower frequency.

MRI is presently extensively used for the investigation of a range of shoulder disorders ${ }^{3}$ and has a high diagnostic accuracy. Interruption of tendon continuity is the most specific MR finding of full thickness rotator cuff tears while subacromial fluid is the most common finding in full thickness rotator cuff tears ${ }^{4}$.

This study was undertaken to comparatively evaluate injuries of the rotator cuff in various population groups using high resolution Ultrasound Imaging System with multifrequency linear array probes and 1.5 Tesla Siemens Symphony MRI equipment. Results of Ultrasound and MRI findings were subsequently compared.

\section{Methods}

50 patients with suspected rotator cuff injuries of the shoulder joint were included in this study over a period of 2 years. The patients had varied presentations like pain in the shoulder joint, traumatic injuries in shoulder joint, restricted and difficulty in movement of shoulder joint. The study was undertaken to correlate the findings on High Resolution Ultrasound and Magnetic Resonance Imaging.

All the cases initially attended the orthopaedic and surgical OPD and were subsequently referred to Radiology Department for further investigation. A detailed history was taken for age, sex, occupation, preexisting illness, cause of pain and mode of injury. Patients were asked about the duration of pain, difficulty with any restriction in shoulder joint movement, inability to bear weight, any weakness and numbness in the arm. This was followed by a thorough clinical examination to determine the site involved, any area of swelling, range of movement to determine the extent of movement restriction, tenderness, lacerations or any associated injuries.

\section{Criteria for Rotator Cuff Tear On High Resolution Ultrasound}

1) Non visualization of the cuff

2) Localized absence or focal non visualization of tendon

3) Discontinuity in tendon

4) Abnormal echogenicity of muscle

\section{MRI Classification for Rotator Cuff Tears}

\begin{tabular}{|c|l|}
\hline Grade & MR Features \\
\hline 0 & Normal- Uniform low signal intensity. \\
\hline 1 & $\begin{array}{l}\text { Diffuse or linear increased signal intensity } \\
\text { on T1WI and PDWI. Normal morphology. }\end{array}$ \\
\hline 2 & $\begin{array}{l}\text { Increased signal intensity on T1WI or PDWI } \\
\text { with thinning or irregularity }\end{array}$ \\
\hline 3 & $\begin{array}{l}\text { Increased signal intensity on T2WI involving } \\
\text { full tendon thickness }\end{array}$ \\
\hline
\end{tabular}

\section{Results}

Fifty patients with history of shoulder pain were evaluated using High Resolution Ultrasonography and then by MRI.

In our study the age incidence ranged from 15 years to 80 years. Mean age of patients was 41.6 years. Most patients (66\%) were between 41-60 years of age. Maximum patients were between $41-50$ years (24\%), followed by $60-80$ years $(22 \%)$ and $51-60$ years $(20 \%)$. The incidence of shoulder injuries was the least $(8 \%)$ in patients below 20 years of age; this was followed by an incidence of $12 \%$ in the age group of 31-40 years.

In our study 14 cases of full thickness tears of the rotator cuff were detected using ultrasonography and MRI. The diagnostic accuracy of ultrasound in detection of complete tear of the supraspinatus muscle was $57.14 \%$ while that of MRI was $100 \%$. Sensitivity and specificity of $\mathrm{MRI}$ in diagnosing full thickness rotator cuff tears in our study are $100 \%$ as compared to those of Evancho et $\mathrm{al}^{5}$ who reported a $80 \%$ sensitivity and $94 \%$ specificity for complete rotator cuff tears by MRI. Burk and coworkers ${ }^{6}$ compared MRI to arthrography, ultrasound, and surgery in a smaller series of surgically confirmed cases. They found that MRI had better sensitivity and specificity $(92 \%$ and $100 \%)$ than ultrasound $(63 \%$ and $50 \%)$ in the detection of full thickness tears. 
In our study the diagnostic accuracy of ultrasound in rotator cuff tears was $57.14 \%$ for full thickness tears and 58.33 for partial thickness tears. The sensitivity and specificity of ultrasound in full thickness tears in our study were $57 \%$ and $50 \%$ respectively and for partial thickness tears were $63.6 \%$ and $60 \%$ respectively. Our result correlates well with the findings of Burk Jr et al (6) in which the reported sensitivity and specificity for ultrasound were $63 \%$ and $50 \%$ respectively. However their study was done in a smaller series of 10 surgically confirmed cases of rotator cuff tears. According to their study large tears were relatively easily detected owing to their marked asymmetry when compared with the normal contralateral side. However small or partialthickness tears in which a defect in the cuff cannot be shown were missed because of the limited contrast resolution of the technique.

In our study, out of the six patients with subacromial/ subdeltoid bursitis five were diagnosed by ultrasound and MRI diagnosed all six. Thus the diagnostic accuracy of ultrasound in detecting subacromial/subdeltoid bursitis

Table 1: Demographic parameters

\begin{tabular}{|l|r|}
\hline Age (Range) & $41.6(15-80)$ \\
Age Distribution & $4(8 \%)$ \\
$<20$ Years & $13(26 \%)$ \\
$20-40$ Years & $33(66 \%)$ \\
$>40$ Years & \\
Sex Distribution & $29(58 \%)$ \\
Male & $21(42 \%)$ \\
Female & \\
Type of lesion & $14(28 \%)$ \\
Full thickness tear of rotator cuff & $12(24 \%)$ \\
Partial thickness tear of supraspinatus & $8(16 \%)$ \\
Subacromial impingement & $5(10 \%)$ \\
Labral tear (SLAP Lesion) & $6(12 \%)$ \\
Subacromial bursitis & $5(10 \%)$ \\
Calcific tendinitis & \\
\hline
\end{tabular}

was $83.33 \%$ and that of MRI was $100 \%$. However subacromial/subdeltoid bursitis in these patients were not associated with rotator cuff tears. According to Teefey et al (9) when fluid is present in both the subacromial/ subdeltoid bursa and the joint, the probability of a rotator cuff tear is $95 \%$. When fluid is present only in bursa, the predictive value for a cuff tear decreases to $70 \%$., and when fluid is seen only in the joint, to $60 \%$. In an another study by Hollister et al (10) who evaluated the association between sonographically detected joint fluid and rotator cuff disease in 163 shoulders, it was found that sonographic finding of intra-articular fluid alone (without bursal fluid) has both a low sensitivity and a low specificity for the diagnosis of rotator cuff tears. However, the finding of fluid in the subacromial/subdeltoid bursa especially when combined with a joint effusion is highly specific and has a high positive predictive value for associated rotator cuff tears. Our findings correlate well with the findings of these two studies.

\section{Discussion}

In the present study 50 patients who presented with clinical signs and symptoms of rotator cuff injuries were subjected to plain radiograph of the affected shoulder followed by high-resolution ultrasound using linear array transducer of multiple frequencies ranging from $7 \mathrm{MHz}$ to $10 \mathrm{MHz}$ and then by MRI using 1.5Tesla equipment. The findings on ultrasonography and MRI were then correlated.

Tears of the subscapularis tendon have been considered uncommon, Codman et $\mathrm{al}^{7}$ reporting an incidence of $3.5 \%$ involvement of the subscapularis in a series of 200 rotator cuff tears. It has been seen that eight percent of rotator cuff injuries have tears of the subscapularis tendon in association with tears of other components of the rotator cuff in a more recent series ${ }^{8}$. Isolated ruptures of the subscapularis tendon are

Table 2: Diagnostic Accuracy of Shoulder Lesions on High Resolution USG and MRI

\begin{tabular}{|l|l|l|l|l|l|}
\hline Type of Lesion & USG & MRI & $\begin{array}{l}\text { Total } \\
\text { Cases }\end{array}$ & $\begin{array}{l}\text { Diagnostic } \\
\text { Accuracy on USG } \\
(\%)\end{array}$ & $\begin{array}{l}\text { Diagnostic Accuracy } \\
\text { on MRI (\%) }\end{array}$ \\
\hline Full Thickness Tear of Rotator cuff & 8 & 14 & 14 & 57.14 & 100 \\
\hline $\begin{array}{l}\text { Partial Thickness Tear of } \\
\text { Supraspinatus }\end{array}$ & 7 & 9 & 12 & 58.33 & 75 \\
\hline Subacromial Impingement & 2 & 7 & 8 & 25 & 87.5 \\
\hline Labral Tears & 2 & 4 & 5 & 40 & 80 \\
\hline Subacromial/Subdeltoid Bursitis & 5 & 6 & 6 & 83.33 & 100 \\
\hline Calcific Supraspinatus Tendinitis & 4 & 5 & 5 & 80 & 100 \\
\hline
\end{tabular}


considered uncommon but may occur secondary to acute abduction and external rotation trauma and in elderly patients with recurrent anterior dislocation ${ }^{8}$. Rupture or subluxation of the biceps tendon is also commonly seen in association with tears of the subscapularis tendon ${ }^{8}$. These findings correlate well with our study in which one case of complete rupture of the subscapularis tendon in association with full thickness tear of supraspinatus and dislocation of the long head of biceps was detected in MRI which could not be seen on ultrasound. Thus the percentage of involvement of subscapularis in our study was $3.8 \%$.

Shoulder impingement is a syndrome where active arm abduction or elevation creates pain as the supraspinatus tendon slides beneath the coracoacromial arch. This can occur when the space containing the supraspinatus bound by the scapula and the coracoacromial arch is decreased, such as from a subacromial entheosphyte spur. The sequelae of shoulder impingement include subacromial-subdeltoid bursitis and rotator cuff tear ${ }^{11}$. Impingement syndrome affects people of all ages from young adults to the elderly and is most often due to repetitive trauma caused by vigorous overhead occupational or athletic endeavors and /or degenerative exostoses ${ }^{12-15}$. With sonography, the diagnosis of shoulder impingement can be suggested when pooling of fluid within the subacromial-subdeltoid bursa occurs with active arm elevation ${ }^{11}$. Out of 50 patients in our study 8 patients had subacromial impingement syndrome. We could confirm 2 patients of impingement syndrome by dynamic ultrasound and the other 7 were confirmed on MRI. The diagnostic accuracy of ultrasound was $25 \%$ and that of MRI $87.5 \%$. The sensitivity and specificity of ultrasound was $25 \%$ and $97.7 \%$, whereas that of MRI was $87.5 \%$ and 95.45 respectively. Farin, Jaroma et al $^{16}$ reported sensitivity of $71 \%$ and specificity of $96 \%$ and an accuracy of $91 \%$ in the ultrasonographic diagnosis of early stage of impingement (stage I-II, without rotator cuff tear). In the present study osteophytes and subacromial spurs were seen in only three cases. In the present study, fluid collection could be observed in two patients, which was detected on shoulder ultrasound. These fluid collections represent lesions defined by $\mathrm{Neer}^{17}$ and Neer and Welsh ${ }^{18}$ as stage I or stage II impingement. MRI is capable of depicting several soft - tissue and bony abnormalities clinically described in impingement syndrome. In regions of inflammation MRI depicted compression of supraspinatus and subacromial bursa by spur in 3 shoulders, capsular hypertrophy of the acromioclavicular joint in 2 shoulders and a low lying acromion in 2 shoulders. While T1 weighted MR imaging is highly sensitive to abnormalities of the supraspinatus tendon, tendonitis could be differentiated from a small tear of the supraspinatus tendon only with T2-weighted imaging.

Ultrasonography can detect labral tears but its diagnostic accuracy is less, being only $40 \%$. Out of the five patients with labral tears in the present study ultrasound detected only two whereas MRI detected 4 patients. Thus MRI had a diagnostic accuracy of $80 \%$. Glenoid labral tears have been associated with overhead throwing activities, trauma, and shoulder instability like recurrent dislocations. All the five patients presented with complaints of acute shoulder pain associated with weakness in abduction and forward flexion. Two patients out of five had recurrent dislocations. Routine shoulder roentgenograms were normal in all those 5 cases of labral tears. The sensitivity of ultrasound in the diagnosis of glenoid labral tears in our study was $50 \%$ whereas sensitivity of ultrasound has ranged from $67 \%$ to $95 \%$ as concluded by Hammar MV et al ${ }^{19}$ and Taljanovic MS et $a^{20}$. Our findings of labral tears in dynamic ultrasound were similar to that as described by Hammar et al $^{19}$ who reported that a hypoechoic zone larger than $2 \mathrm{~mm}$ or abnormal labral movement with dynamic imaging indicates labral tear. The sensitivity and specificity of MRI in diagnosing labral tears in our study were $80 \%$ and $94 \%$ respectively by using a combination of gradient-echo and high-resolution fastspin-echo axial pulse sequences. Gusmer PB, Potter $\mathrm{HG}$ et $\mathrm{a}^{21}$ carried out an unenhanced MR imaging in one hundred three patients with clinically suspected shoulder injuries and prospectively assessed the accuracy of magnetic resonance (MR) imaging in the detection of labral injuries using same pulse sequences as in our study. Surgical correlation was obtained in all patients. The sensitivity and specificity for detection of these tears with MR imaging was $86 \%$ and $100 \%$ respectively. Overall MR imaging was $95 \%$ accurate in the detection of labral injuries in their study.

A total of five cases of calcific supraspinatus tendonitis were detected in our study of patients with painful shoulders. Patients presented with symptoms of pain at rest, at night and limitation of motion of the shoulder joint. The age range of the patients affected with calcific supraspinatus tendinitis was from 40 to 70 years. The pathogenesis of calcium hydroxyapatite crystal deposition is unknown, although trauma, ischaemia, or other systemic factors may induce abnormalities in the connective tissue, leading to crystal deposition ${ }^{22,23}$. 
Crystal deposition most commonly occurs in the tendinous and bursal structures about the shoulder, particularly the supraspinatus tendon. Gotoh $\mathrm{M}$ et $\mathrm{al}^{24}$ in 2003 documented the clinical, radiographic and histologic findings in a 46-year-old man with calcifying tendinitis in his left shoulder, which progressed to rotator cuff tear. The patient had a one-year history of repeated calcifying tendinitis before being referred to the hospital. Initial radiographs and magnetic resonance imaging (MRI) revealed calcium deposition localized in the supraspinatus tendon without apparent tear. MRI done after 3 months revealed a partial-thickness rotator cuff tear at the site of calcium deposition. Surgical and histologic findings demonstrated that calcium deposition was the cause of cuff rupture. According to the authors this was the first case based on review of literature so far in which the progression from calcifying tendinitis to rotator cuff tear was serially observed. The five cases of calcific supraspinatus in our study however were lost in follow up.

Thus in the evaluation of rotator cuff injuries high resolution ultrasonography is the primary imaging modality, however MRI continues to remain the gold standard in accurate localization, characterization and assessment of rotator cuff injuries.

\section{Conclusion}

MRI however a costly investigation, is the gold standard in evaluation of rotator cuff pathologies. Ultrasound although being operator dependent and not as accurate as MRI, provides a rapid real time non-invasive cross sectional imaging of the joint. High resolution Ultrasound can be considered as primary modality for imaging rotator cuff pathologies, as it is readily available and is cost effective.

\section{References}

1. Charles AR, Frederick AM. The Shoulder- Volume 2, 650-52.

2. Carol MR, Stephanie RW, William C. Diagnostic Ultrasound, Volume One,Second Edition, 1998. 843-844.

3. Blanchard T, Mackenzie R, Bearcroft P, Sinnatamby R, Gray A. Magnetic Resonance Imaging of the Shoulder: Assessment of Effectiveness. Clinical Radiology 1997. 52: 363-68.

4. Farley T.E, Neumann C.H, Steinback L S, Jahnke A.J., Peterson S.S. Full Thickness Tears of the
Rotator Cuff of the Shoulder: Diagnosis with MR Imaging. AJR 1992. 158 : 347-351.

5. Evancho AM, Stiles RG, Fajiman WA: MR imaging diagnosis of rotator cuff tears, AJR1988: 151: 751754.

6. DL Burk, Jr, D Karasick, A B.Kurtz, DG Mitchell, MD Rifkin, CL Miller, DW Levy, JM Fenlin, AR BarolozziRotator Cuff Tears: Prospective Comparison of MRImaging with Arthrography, Sonography, and Surgery.AJR 1989. 153: 87-92.

7. Codman EA. The shoulder: rupture of the supraspinatus tendon and other lesions in or about the subacromial space. Boston: Thomas Todd: 1934.

8. Deutsch A, Altchek DW, Veltri DM, et al. Traumatic tears of the subscapularis tendon: Clinical diagnosis, magnetic resonance imaging findings, and operative treatment. Am J Sports Med 1997; 25: 13-22.

9. William D. Middleton, Sharlene A. Teefey, Ken Yamaguchi: Sonography of the rotator cuff: Analysis of Interobserver Variability. AJR 2004: 183: $1465-1468$.

10. Mary S. Hollister, Mack L A, Patten RM, Winter TC, Matsen FA. Veith RR. Association of Sonographically Detected Subacromial and Subdeltoid Bursal Effusion and Intra articular fluid with Rotator Cuff Tear. AJR 1995. 165; 3: 605-608.

11. Farin PU, Jaroma H, Harju A, Soimakallio S. Shoulder impingement Syndrome: sonographic evaluation. Radiology 1990; 176: 845-9.

12. Penny JN. Welsh MB. Shoulder impingement syndromes in athletes and their surgical management. Am J Sports Med 1981: 9: 11-15

13. Neer CS II. Anterior acromioplasty for the chronic impingement syndrome in the shoulder. J Bone Joint Surg (Am) 1972; 54-A: 41-50.

14. Neer CS II. Impingement lesions. Clin Orthop 1983: 173: $70-77$.

15. Cone RO III, Resnick D, Danzig L. Shoulder impingement syndrome: radiographic evaluation. Radiology 1984; 150: 29-33.

16. Pekka U. Farin, Heikki Jaroma et al : Shoulder Impingement Syndrome: Sonographic Evaluation: Radiology 1990; 176: 845-849. 
17. Neer CS II. Impingement lesions. Clin Orthop 1983: 173: 70-77.

18. Neer CS II. Welsh RP. The shoulder in sports. Orthop Clin North Am 1977: 8: 583-591.

19. Hammar MV, Wintzell GB, Astorm KGO, Larsson S, Elvin A. Role of US in the preoperative evaluation of patients with anterior shoulder instability.Radiology 2001; 19: 29-34.

20. Taljanovic MS, Carlson KL, Kuhn JE, Jacobson JA, Delaney -Sathy LO, Adler RS. Sonography of the glenoid labrum: a cadaveric study with arthroscopic correlation.AJR Am J Roengenol 2000; 174: 171722.
21. Gusmer PB, Potter HG, Schatz JA, Wickiewicz TL et al; Labral injuries: accuracy of detection with unenhanced MR imaging of the shoulder: Radiology.1996 Aug; 2002: 519-24.

22. Greenway GD, Danzig LA, Resnick D, Haghighi P. The painful shoulder. Med Radiogr Photogr 1982; 58: 22-67.

23. Zlatkin MB, Dalinka MKD, Crystal deposition diseases: current concepts. Postgra Radiol 1988; 8: 88-98.

24. Gotoh M, Higuchi F, Suzuki R, Yamanaka K. Progression from calcifying tendinitis to rotator cuff tear. Skeletal Radiol : 2003 Feb; 32(2) : 86-9. 